\title{
Erratum: Boundary and interface CFTs from the conformal bootstrap
}

\author{
Ferdinando Gliozzi, ${ }^{a, b}$ Pedro Liendo, ${ }^{c}$ Marco Meineri ${ }^{d, e}$ and Antonio Rago ${ }^{f}$ \\ ${ }^{a}$ Dipartimento di Fisica, Università di Torino, \\ Via P. Giuria 1 I-10125 Torino, Italy \\ ${ }^{b}$ Istituto Nazionale di Fisica Nucleare - Sezione di Torino, \\ Via P. Giuria 1 I-10125 Torino, Italy \\ ${ }^{c} I M I P$, Humboldt-Universität zu Berlin, IRIS Adelershof, \\ Zum Großen Windkanal 6, 12489 Berlin, Germany \\ ${ }^{d}$ Scuola Normale Superiore, \\ Piazza dei Cavalieri 7 I-56126 Pisa, Italy \\ e Istituto Nazionale di Fisica Nucleare - Sezione di Pisa, \\ Largo B. Pontecorvo, 3, 56127 Pisa, Italy \\ ${ }^{f}$ Centre for Mathematical Sciences, Plymouth University, \\ Drake Circus, Plymouth, PL4 8AA, U.K. \\ E-mail: ferdinando.gliozzi@to.infn.it, pliendo@physik.hu-berlin.de, \\ marco.meineri@sns.it, antonio.rago@plymouth.ac.uk
}

ERratum to: JHEP05(2015)036

ArXIV EPRINT: 1502.07217

Equations (3.8)-(3.11) in the published version of this paper report results about the extraordinary transition for $N>1$. However, the fusion rule in the boundary channel was incorrectly assumed to only include the identity, the displacement operator and higher dimensional primaries. Instead, a protected boundary operator of dimension $\widehat{\Delta}=2$ arises from the breaking of the continuous $O(N)$ symmetry. Hence, the results in question should be disregarded. We refer to [1] for a conformal bootstrap study of the extraordinary transition for $N>1$.

Open Access. This article is distributed under the terms of the Creative Commons Attribution License (CC-BY 4.0), which permits any use, distribution and reproduction in any medium, provided the original author(s) and source are credited.

\section{References}

[1] J. Padayasi, A. Krishnan, M. A. Metlitski, I. A. Gruzberg and M. Meineri, The extraordinary boundary transition in the $3 d O(N)$ model via conformal bootstrap, arXiv:2111.03071. 